\title{
Mitglieder können die Bezugsart ab sofort frei wählen
}

Bisher erhalten Mitglieder der DEGUM die Zeitschrift „Ultraschall in der Medizin“ (UiM) als Print- und Online-Version. Der Zugang zur Online-Version befindet sich im internen Bereich der DEGUM-Homepage.

Am 25. September 2015 entschied die DEGUM-Mitgliederversammlung, dass jedes Mitglied ab 1. Januar 2016 selbst entscheiden kann, wie es die Zeitschrift künftig bezieht: entweder print und online oder nur online.
Mitglieder, die sich entscheiden, ab 2016 die Zeitschrift nur noch online zu beziehen, und diesen Wunsch noch nicht mitgeteilt haben, wenden sich bitte an die Geschäftsstelle der DEGUM.

Für Mitglieder, die die UiM weiterhin in gedruckter Form sowie online beziehen möchten, besteht kein Handlungsbedarf.

Der veränderte Bezugspreis für OnlineOnly wird aktuell nicht weitergereicht. Auf der Mitgliederversammlung in Leip- zig 2016 wird über die weitere Verwendung der eingesparten Kosten entschieden.

Bei Fragen steht die Geschäftsstelle der DEGUM gerne zur Verfügung. 